\title{
Asociación entre malnutrición por exceso con caries temprana de la infancia
}

\author{
CARLOS ZAROR S. ${ }^{1,2}$, JORGE SAPUNAR Z. ${ }^{2}$, \\ SERGIO MUÑOZ N. ${ }^{3}$, DAMARIS GONZÁLEZ C. ${ }^{4}$ \\ 1. Departamento de Odontopediatría y Ortodoncia, Facultad de Odontología. Universidad de La Frontera, Temuco. \\ 2. Centro de Excelencia CIGES, Facultad de Medicina, Universidad de la Frontera. \\ 3. Departamento de Salud Pública, Facultad de Medicina, Universidad de La Frontera, Temuco. \\ 4. Servicio de Salud del Reloncaví, X Región, Chile.
}

\begin{abstract}
Association between overweight and early childhood caries

Objective: To determine whether overweight is a risk factor for the development of early childhood caries (ECC) in preschoolers. Patients and Method: An observational retrospective cohort study was performed in 196 children under 2 years of age at Calbuco Hospital, Los Lagos Region, Chile, who were admitted between 2007 and 2009. Patients were grouped based on their nutritional status at admission in the two following categories: eutrophic and overweighed children as a result of excessive intake. Information regarding caries incidence and nutritional status were annually retrieved from records until the age of 5 years, according to medical and dental records. Results: At the beginning of the study, $33.67 \%$ were overweight and $16.33 \%$ were obese, reaching $40 \%$ overweight and $20.56 \%$ obese at four years of age. The incidence of early childhood caries in overweighed children was $57.14 \%$ compared to $40.82 \%$ in normal weight children $(\mathrm{p}=0.022)$, with 1.4 RR (95\% CI, 1044-1.88). Conclusions: The increased risk of early childhood decay in overweighed patients makes necessary to create instances of timely mutual referral between the professional that diagnoses overweighed children and the pediatric dentist in order to develop preventive treatments for both diseases.
\end{abstract}

(Key words: Dental caries, obesity, overweight).

Rev Chil Pediatr 2014; 85 (4): 455-461

\section{RESUMEN}

Objetivo: Determinar si la malnutrición por exceso es un factor de riesgo para el desarrollo de caries temprana de la infancia (CTI) en preescolares. Pacientes y Método: Se realizó un estudio observacional de cohorte retrospectivo en 196 niños de 2 años del Hospital de Calbuco, Región de Los Lagos, Chile, ingresados durante los años 2007 a 2009. Los pacientes fueron agrupados de acuerdo a su estado nutricional al ingreso en niños eutróficos y niños con malnutrición por exceso. La información respecto a incidencia de caries y el estado

Recibido el 24 de agosto de 2013, última versión aceptada para publicación el 02 de abril de 2014.

Potenciales conflictos de interés: Este trabajo cumple con los requisitos sobre consentimiento /asentimiento informado, comité de ética, financiamiento, estudios animales y sobre la ausencia de conflictos de intereses según corresponda.

Correspondencia a:

Carlos Zaror Sánchez

E-mail: carlos.zaror@ufrontera.cl 
nutricional fueron recuperados de la ficha anualmente hasta la edad de 5 años, según registro de las fichas médicas y odontológicas. Resultados: El 33,67\% estaba en sobrepeso al inicio de la cohorte y el 16,33\% obeso, alcanzando $40 \%$ sobrepeso y $20,56 \%$ obesidad a los cuatro años de edad. La incidencia de caries temprana de la infancia en niños con malnutrición por exceso fue de $57,14 \%$ en relación al $40,82 \%$ de los niños eutróficos $(\mathrm{p}=0,022)$ con RR de 1,4 (95\% IC, 1.044-1,88). Conclusiones: El mayor riesgo asociado a CTI en pacientes con malnutrición por exceso invita a generar las instancias de derivación oportuna desde el profesional que pesquisa a niños con malnutrición por exceso hacia el odontólogo infantil, con el fin de desarrollar intervenciones preventivas para ambas patologías.

(Palabras clave: Caries, dental, obesidad, sobrepeso).

Rev Chil Pediatr 2014; 85 (4): 455-461

\section{Introducción}

La Caries Temprana de la Infancia (CTI) es una forma severa y particular de caries, de carácter multifactorial, que afecta la dentición primaria de lactantes y niños pre-escolares ${ }^{1}$. Constituye un serio problema de salud pública a nivel mundial, siendo más prevalente en países en vías de desarrollo y subgrupos desprotegidos de países industrializados como inmigrantes, minorías étnicas o comunidades rurales, en quienes la prevalencia alcanza hasta un $70 \%{ }^{2}$. En Chile, la prevalencia comunicada varía desde un $7,8 \%$ a un $88 \%$. El último reporte ministerial, en el año 2007, reveló una prevalencia de un $27 \%$ a los 2 años y de un $48 \%$ a los 4 años ${ }^{3}$.

Aún cuando la etiología de CTI es multifactorial, el principal factor etiopatogénico es la fermentación de hidratos de carbono por parte del streptococcus mutans, constituyente del biofilm bacteriano ${ }^{4}$, mecanismo favorecido por la ingesta excesiva de hidratos de carbono fermentables atribuible a factores culturales, socio-económicos y conductuales ${ }^{5,6}$.

La malnutrición por exceso se define como el aumento en la adiposidad por aporte excesivo de nutrientes en relación a las necesidades metabólicas. Esta condición, que incluye el sobrepeso y la obesidad, ha experimentado un notable aumento en su frecuencia los últimos 15 años $^{7}$. En Chile la prevalencia de sobrepeso y obesidad infantil también ha aumentado de forma importante. La obesidad afectaba al $8,6 \%$ de los niños entre 2 y 5 años el año $2002^{8}$. En el año 2007 la prevalencia de obesidad varió entre $11 \%$ y $20 \%$ en niños del mismo grupo etario?.
Una de las características de la malnutrición por exceso en niños es la ingesta preferente de hidratos de carbono fermentables, aspecto que podría vincular al trastorno nutricional con $\mathrm{CTI}^{10}$. Existe fuerte evidencia que soporta la asociación entre CTI y dietas ricas en carbohidratos refinados y entre ésta con obesidad en niños ${ }^{11,12}$. Por lo tanto, resulta biológicamente posible la relación entre caries dental y estado nutricional. Sin embargo, la evidencia no es concluyente en establecer si existe asociación entre caries dental y peso o si ambas patologías sólo coexisten al tener factores etiológicos comunes como son la dieta y el nivel socioeconómico ${ }^{13}$.

Dada la falta de consenso en la literatura biomédica y la ausencia de estudios en nuestro país, el objetivo principal de esta investigación es determinar la asociación entre caries dental y malnutrición por exceso en prescolares chilenos de nivel socioeconómico medio-bajo.

Se establecieron como objetivos específicos el determinar: características socio-demográficas de la muestra, prevalencia de obesidad y sobrepeso al inicio, incidencia de CTI, promedio de índice ceod, incidencia de CTI según estado nutricional, y distribución de las variables de control (género, presencia de higiene, índice higiene oral, uso mamadera, uso mamadera nocturna, lactancia materna prolongada, historia de caries) según incidencia de CTI y promedio del índice ceod.

\section{Pacientes y Método}

\section{Diseño}

Se realizó un estudio observacional de cohorte retrospectivo para determinar si la mal- 
nutrición por exceso está asociada con el desarrollo de CTI. La investigación se realizó en el Hospital de Calbuco, perteneciente al Servicio de Salud del Reloncaví, región de Los Lagos, Chile. Calbuco es un archipiélago de $30 \mathrm{mil}$ habitantes, principalmente de nivel socioeconómico medio-bajo y con un alto índice de ruralidad.

\section{Pacientes}

Se incluyeron en el estudio todos los niños de 2 años ingresados al Hospital de Calbuco durante los años 2007 a 2009. Se excluyeron niños con discapacidad o enfermedad sistémica de base, niños con malnutrición por carencia, con caries cavitadas y/o con un período de seguimiento menor a un año.

Los pacientes fueron agrupados de acuerdo a su estado nutricional al ingreso en niños eutróficos y con malnutrición por exceso.

Se extrajo de la ficha odontológica todos los datos socio-demográficos, información sobre hábitos dietarios e higiénicos, incidencia de caries y el índice ceod que mide la experiencia o historia de caries en dentición primaria mediante el número de dientes cariados, obturados o con indicación de extracción por caries.

La incidencia de caries se estableció en base a los criterios propuestos por la OMS en el Oral Health Survey Basic Methods, para estudios epidemiológicos ${ }^{14}$ y la higiene oral se determinó mediante el índice de Higiene Oral Simplificado de Greene y Vermillion (IHOS) ${ }^{15}$.

De la ficha médica se obtuvieron los datos de salud general del niño y la antropometría con el fin de determinar su estado nutricional inicial y el de cada control. El estado nutricional se determinó de acuerdo a las referencias de la OMS para la evaluación antropométrica de niños menores de 6 años, adoptadas por el Ministerio de Salud de Chile ${ }^{16}$.

La incidencia de caries y el estado nutricional fueron analizados anualmente hasta la edad de 5 años, según registro de las fichas médicas y odontológicas.

\section{Análisis estadístico y tamaño muestral}

Se estimó un tamaño muestral mínimo de 92 niños por grupo, considerando una prevalencia de CTI del $84 \%$ en obesos y $65 \%$ en no obesos de acuerdo a los resultados de un estudio de prevalencia realizado en la misma población ${ }^{3}$, con un nivel de significancia de un $5 \%$ y una potencia estadística de un $80 \%$.

El análisis estadístico de los datos y el cálculo de tamaño muestral se llevó a cabo por medio del programa Stata 10 (Stata Corp LP, USA). La asociación independiente entre las variables de respuesta con las variables de exposición y de control se determinó mediante el test exacto de Fisher para la variable de respuesta dicotómica presencia de caries. Para las variables de respuesta continua índice ceod se utilizó la prueba ttest para varianzas no homogéneas. En ambos casos se consideró un nivel de significación de 0,05. El análisis inferencial se generó mediante un modelo de regresión poisson modificada ${ }^{17}$ que incluyó las variables de interacción establecidas mediante el análisis estratificado, con un nivel de confianza de un $95 \%$.

\section{Resultados}

La muestra la constituyeron 196 niños de 2 años, de los cuales el 50\% (98/196) correspondió a individuos de sexo masculino. Un $69,39 \%(136 / 196)$ perteneció a zonas urbanas y un 50,51\% (98/196) tenía previsión nivel A del Fondo Nacional de Salud.

Al inicio del estudio el 33,67\% (66/196) tenía sobrepeso y el 16,33\% (32/196) obesidad. $\mathrm{Al}$ aumentar la edad de los sujetos los trastornos nutricionales aumentaron su frecuencia hasta alcanzar $60,56 \%(109 / 180)$ a los cuatro años de edad (figura 1).

La tabla 1 detalla la distribución de la muestra según hábitos alimenticios e higiénicos, las cuales fueron medidas al inicio del estudio.

El índice ceod promedio inicial fue de $1,1 \pm 2,39$. La incidencia de caries temprana de la infancia fue de un 48,98\% (96/196) con un ceod promedio de 2,9 $\pm 3,56$ al final del seguimiento. El período de seguimiento promedio fue de $21 \pm 8,8$ meses.

Los niños que sufrían de malnutrición por exceso presentaron una incidencia de CTI de $57,14 \%$ (56/98) en comparación al 40,82\% (40/98) que padecieron los niños con relación peso/talla normal (figura 2), diferencia que fue 


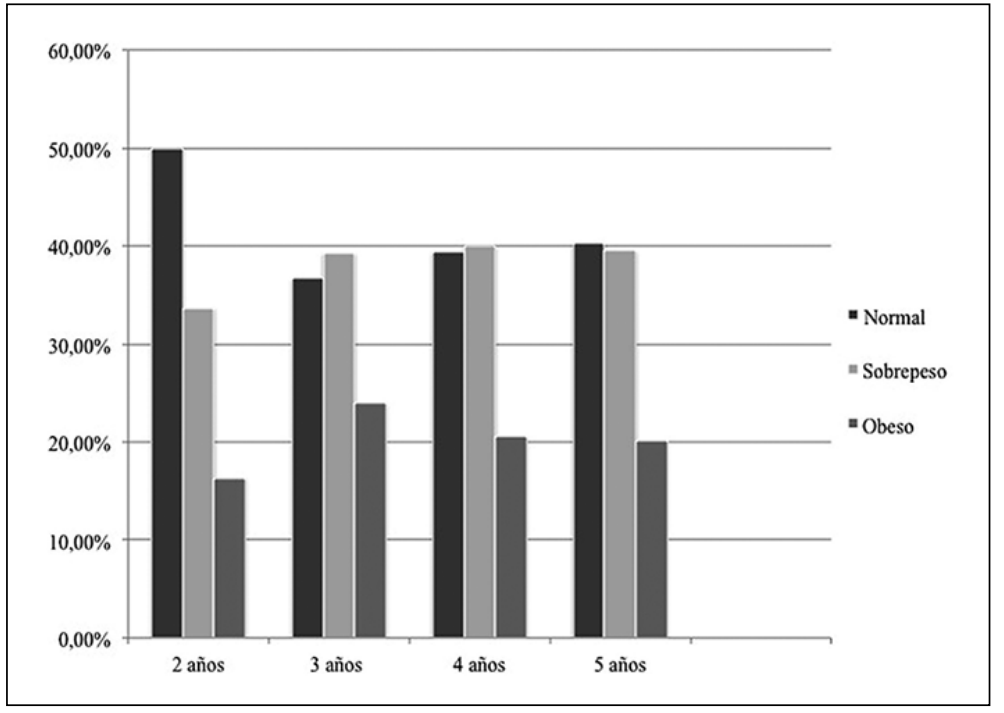

Figura 1. Estado nutricional a las diferentes edades.

Tabla 1. Descripción de la muestra según hábitos alimenticios e higiénicos

\begin{tabular}{|c|c|c|}
\hline Característica & Frecuencia & Porcentaje \\
\hline $\begin{array}{l}\text { Lactancia materna } \\
\text { No prolongada ( } \leq 12 \text { meses) } \\
\text { Prolongada ( }>12 \text { meses) }\end{array}$ & $\begin{array}{r}101 \\
94\end{array}$ & $\begin{array}{l}51,79 \\
48,21\end{array}$ \\
\hline $\begin{array}{l}\text { Uso de mamadera } \\
\text { Ausente } \\
\text { Presente }\end{array}$ & $\begin{array}{r}34 \\
162\end{array}$ & $\begin{array}{l}17,35 \\
82,65\end{array}$ \\
\hline $\begin{array}{l}\text { Uso mamadera nocturna } \\
\text { Ausente } \\
\text { Presente }\end{array}$ & $\begin{array}{r}120 \\
75\end{array}$ & $\begin{array}{l}61,54 \\
38,46\end{array}$ \\
\hline $\begin{array}{l}\text { Higiene oral } \\
\text { Ausente } \\
\text { Presente }\end{array}$ & $\begin{array}{r}18 \\
178\end{array}$ & $\begin{array}{r}9,18 \\
90,82\end{array}$ \\
\hline $\begin{array}{l}\text { Cepillado nocturno } \\
\text { Ausente } \\
\text { Presente }\end{array}$ & $\begin{array}{r}132 \\
64\end{array}$ & $\begin{array}{l}67,35 \\
32,65\end{array}$ \\
\hline $\begin{array}{l}\text { Índice higiene oral simplificado } \\
\text { Adecuado } \\
\text { Deficiente }\end{array}$ & $\begin{array}{r}14 \\
128\end{array}$ & $\begin{array}{r}9,86 \\
90,14\end{array}$ \\
\hline
\end{tabular}

estadísticamente significativa $(p=0,022)$. El riesgo relativo de caries fue de 1,4 (95\% IC, 1.044-1,878), por lo tanto, hubo 1,40 veces más probabilidad de sufrir nuevas lesiones de caries en pacientes que presenten sobrepeso $\mathrm{u}$ obesidad en relación a los individuos que presentan un peso adecuado para su talla.

La asociación entre incidencia de CTI y las variables de control se detalla en la tabla 2 .
Tabla 2. Asociación entre incidencia de Caries Temprana de la Infancia (CTI) con variables de control

\begin{tabular}{|c|c|c|c|c|}
\hline \multirow[t]{2}{*}{ Variable } & \multicolumn{4}{|c|}{ Incidencia CTI } \\
\hline & Total & $\mathbf{n}$ & $\%$ & $\mathbf{p}$ \\
\hline $\begin{array}{l}\text { Género } \\
\text { Masculino } \\
\text { Femenino }\end{array}$ & $\begin{array}{l}98 \\
98\end{array}$ & $\begin{array}{l}53 \\
43\end{array}$ & $\begin{array}{l}54,08 \\
43,88\end{array}$ & 0,153 \\
\hline $\begin{array}{l}\text { Residencia } \\
\text { Rural } \\
\text { Urbano }\end{array}$ & $\begin{array}{r}60 \\
136\end{array}$ & $\begin{array}{l}34 \\
62\end{array}$ & $\begin{array}{l}56,57 \\
45,59\end{array}$ & 0,198 \\
\hline $\begin{array}{l}\text { Previsión } \\
\text { FONASA A } \\
\text { FONASA B - C - D }\end{array}$ & $\begin{array}{l}99 \\
97\end{array}$ & $\begin{array}{l}56 \\
40\end{array}$ & $\begin{array}{l}56,57 \\
41,24\end{array}$ & $0,034^{*}$ \\
\hline $\begin{array}{l}\text { Historia de caries } \\
\text { Ausente } \\
\text { Presente }\end{array}$ & $\begin{array}{r}144 \\
52\end{array}$ & $\begin{array}{l}54 \\
42\end{array}$ & $\begin{array}{l}37,50 \\
80,77\end{array}$ & $\underset{0,001 *}{<}$ \\
\hline $\begin{array}{l}\text { Lactancia materna } \\
\text { No prolongada ( } \leq 12 \text { meses) } \\
\text { Prolongada ( }>12 \text { meses) }\end{array}$ & $\begin{array}{r}101 \\
94\end{array}$ & $\begin{array}{l}38 \\
57\end{array}$ & $\begin{array}{l}37,62 \\
60,64\end{array}$ & $0,002^{*}$ \\
\hline $\begin{array}{l}\text { Uso de mamadera } \\
\text { Ausente } \\
\text { Presente }\end{array}$ & $\begin{array}{r}34 \\
162\end{array}$ & $\begin{array}{l}20 \\
76\end{array}$ & $\begin{array}{l}58,82 \\
46,91\end{array}$ & 0,258 \\
\hline $\begin{array}{l}\text { Uso mamadera nocturna } \\
\text { Ausente } \\
\text { Presente }\end{array}$ & $\begin{array}{r}120 \\
75\end{array}$ & $\begin{array}{l}63 \\
32\end{array}$ & $\begin{array}{l}52,50 \\
42,67\end{array}$ & 0,189 \\
\hline $\begin{array}{l}\text { Higiene oral } \\
\text { Ausente } \\
\text { Presente }\end{array}$ & $\begin{array}{r}18 \\
178\end{array}$ & $\begin{array}{l}12 \\
84\end{array}$ & $\begin{array}{l}66,67 \\
47,19\end{array}$ & 0,141 \\
\hline $\begin{array}{l}\text { Cepillado nocturno } \\
\text { Ausente } \\
\text { Presente }\end{array}$ & $\begin{array}{r}132 \\
64\end{array}$ & $\begin{array}{l}68 \\
28\end{array}$ & $\begin{array}{l}51,52 \\
43,75\end{array}$ & 0,361 \\
\hline $\begin{array}{l}\text { Índice higiene oral simplificado } \\
\text { Adecuado } \\
\text { Deficiente }\end{array}$ & $\begin{array}{r}14 \\
128\end{array}$ & $\begin{array}{r}5 \\
66\end{array}$ & $\begin{array}{l}35,71 \\
51,56\end{array}$ & 0,399 \\
\hline
\end{tabular}

*Asociación estadísticamente significativa test exacto de Fisher. 


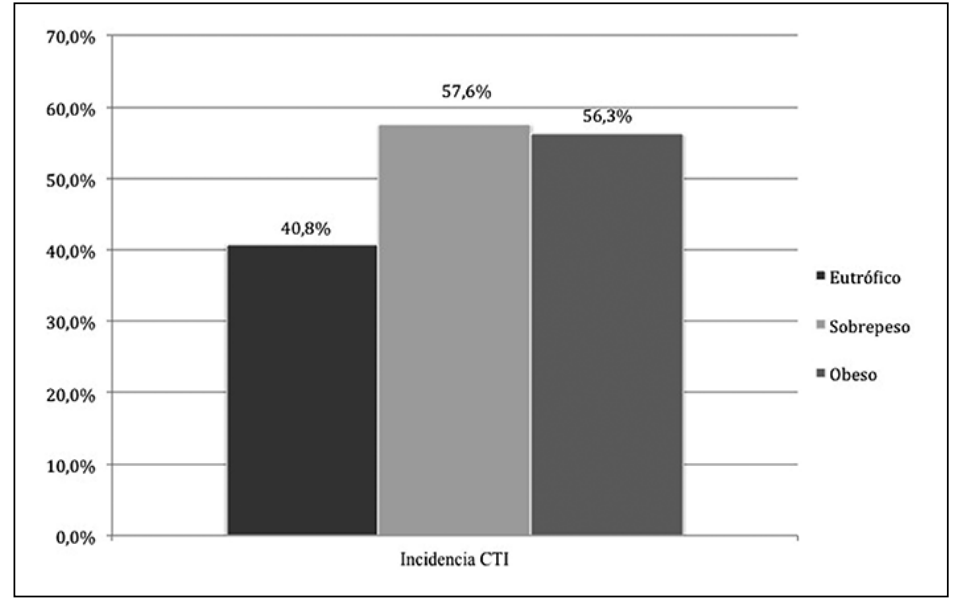

Figura 2. Incidencia de caries según estado nutricional.
Los resultados del estudio mostraron asociación significativa con CTI para las variables previsión, lactancia materna prolongada e historia de caries.

Los niños con historia de caries presentaron un índice ceod promedio de 6,58 $\pm 3,48$ en relación a los niños que no manifestaron lesiones de caries antes del inicio del estudio con un índice ceod promedio de $1,60 \pm 2,52(\mathrm{p}<0,001)$. También se encontró asociación estadísticamente significativa entre índice ceod con previsión, lactancia materna prologada e IHOS $(\mathrm{p}<0,05)$.

Para evaluar la presencia de interacciones se realizó análisis estratificado con las variables de control que dieron significación estadística; previsión, historia de caries, lactancia materna prolongada. Además se incluyeron las variables sexo e higiene oral por encontrarse clínicamente significativas. Se aplicó un nivel de significación de un $10 \%$ para pruebas de homogeneidad.

El género y la higiene mostraron ser modificadores de efecto en donde los preescolares hombres con malnutrición por exceso mostraron 1,8 más probabilidad de tener CTI y los niños a los que no se les realizaba higiene oral presentaron 2,5 veces más probabilidad de presentar CTI (tabla 3).

Se realizó un modelamiento estadístico que incluyó las variables de interacción sexo e higiene. Al ajustar por estas variables el riesgo relativo obtenido fue de 1,49 (95\% IC, 0,397 $5,55)$.
Tabla 3. Riesgo relativo de Caries Temprana de la Infancia (CTI) según trastorno nutricional ajustado por variables de control

\begin{tabular}{|c|c|c|}
\hline Variable & RR (IC) & $\begin{array}{c}\mathbf{p}^{*} \\
\text { homogeneidad }\end{array}$ \\
\hline $\begin{array}{l}\text { Género } \\
\text { Masculino } \\
\text { Femenino }\end{array}$ & $\begin{array}{l}1,79(1,20-2,66) \\
1,05(0,67-1,64)\end{array}$ & $0,079 *$ \\
\hline $\begin{array}{l}\text { Previsión } \\
\text { FONASA A } \\
\text { FONASA B - C - D }\end{array}$ & $\begin{array}{l}1,03(0,92-1,86) \\
1,53(0,94-2,50)\end{array}$ & 0,603 \\
\hline $\begin{array}{l}\text { Historia de caries } \\
\text { Ausente } \\
\text { Presente }\end{array}$ & $\begin{array}{l}1,45(0,93-2,24) \\
1,53(1,17-1,99)\end{array}$ & 0,803 \\
\hline $\begin{array}{l}\text { Lactancia materna } \\
\text { No prolongada } \\
\text { ( } \leq 12 \text { meses) } \\
\text { Prolongada } \\
\text { (>12 meses) }\end{array}$ & $\begin{array}{l}1,63(0,97-2,74) \\
1,23(0,88-1,71)\end{array}$ & 0,357 \\
\hline $\begin{array}{l}\text { Higiene oral } \\
\text { Presente } \\
\text { Ausente }\end{array}$ & $\begin{array}{l}1,30(0,95-1,79) \\
2,50(1,17-5,34)\end{array}$ & $0,119^{*}$ \\
\hline
\end{tabular}

*Asociación estadísticamente significativa prueba de homogeneidad.

\section{Discusión}

Evidencia reciente muestra la importancia de la salud oral en el manejo de la salud sistémica. Caries en particular ha sido asociada a síndromes como xerostomía, patología endocrina o consumo de medicamentos entre otros ${ }^{18,19}$.

Tanto la caries temprana de la infancia como la malnutrición por exceso se caracterizan por compartir similares factores de riesgo, como son el tener pobres hábitos dietéticos $\mathrm{y} / \mathrm{o}$ 
un nivel socioeconómico bajo. Bajo esta premisa, es que el sobrepeso y la obesidad han sido sugeridos como predictores de desarrollo y progresión de la caries dental $1^{13,20,21}$.

Sin embargo, actualmente, no hay consenso en la literatura internacional sobre la relación entre malnutrición por exceso y caries temprana de la infancia ${ }^{11,18,21-26}$. Una revisión sistemática de la literatura evaluó la asociación entre obesidad infanto-juvenil y del adulto con la prevalencia de caries dental. Sus resultados fueron contradictorios por baja calidad metodológica de los estudios incluidos. Sólo un estudio de buena calidad mostró una asociación directa entre obesidad y caries dental ${ }^{27}$. Nuestro estudio al igual que este último, encontró una asociación estadísticamente significativa con un riesgo relativo crudo de 1,40 (95\% IC, 1,044-1,878).

Es importante resaltar que los resultados de nuestro estudio mostraron que esta asociación puede ser modulada por otros factores, como son el sexo y la higiene oral, los cuales pueden modificar la fuerza de esta relación.

La prevalencia de sobrepeso y obesidad en nuestro estudio $(33,67 \%$ y $16,33 \%$ respectivamente) duplica la media nacional reportada por el MINSAL en el $2002^{8}$.

$\mathrm{Al}$ asociar CTI con las diferentes variables de control en estudio sólo encontramos asociación con nivel de previsión, historia de caries y lactancia materna prolongada. Esto es confirmado por la evidencia existente sobre todo para las dos primeras variables, en donde la incidencia de CTI encontrada $(48,98 \%)$ concuerda con las prevalencias encontradas en otros estudios epidemiológicos realizados en países en vías de desarrollo y poblaciones desfavorecidas de países desarrollados 5,6 . Marshall et al. ${ }^{13}$, evaluaron la asociación entre caries y obesidad y cómo el rol de la dieta junto con en el nivel socioeconómico influyen en esta relación. En su estudio concluyeron que tanto la caries como la obesidad coexisten en niños de bajo nivel socioeconómico, por lo tanto, las medidas de salud pública deberían estar orientadas a un adecuado asesoramiento dietético junto con el acceso a una dieta saludable con el fin de disminuir el riesgo de ambas enfermedades en esta población.

Esto también es reforzado por Oliveira et al. ${ }^{28}$, que también encontraron relación entre factores nutricionales y socioeconómicos con caries dental en preescolares.

La experiencia pasada de caries es un importante predictor de nuevas lesiones de caries como lo confirman Li y Wang en el $2002^{29}$, mediante un estudio de cohorte de 8 años. Ellos concluyeron que la existencia de caries en la dentición primaria puede ser un importante indicador de riesgo para predecir caries en la dentición permanente.

En el caso de la lactancia materna prolongada la literatura es controversial. Si bien algunos autores han relacionado a la CTI con la lactancia materna prolongada más allá del año de vida $^{1}$, otros como Ribeiro ${ }^{30}$ presentan una serie de argumentos que refutan la asociación entre lactancia materna y CTI.

Lo que está claro es que la relación entre caries y dieta es compleja y que hay muchas variables biológicas tales como infección de streptococcus mutans, hipoplasia de esmalte, consumo de azúcar, así como también variables como nivel educacional de la madre y/o estado socioeconómico que pueden influir y afectar la salud oral.

La principal limitación de nuestro estudio radica en la imposibilidad de analizar la variabilidad de las variables de control en el tiempo, por lo que sólo se controló considerando que las variables se mantenían igual durante todo el período de seguimiento. Esto sugiere realizar estudios prospectivos, que permitan medir los posibles cambios de las variables que intervienen en el desarrollo de la caries temprana de la infancia y su asociación con malnutrición por exceso.

El mayor riesgo asociado a CTI en pacientes con malnutrición por exceso invita a generar las instancias de derivación oportuna desde el profesional que pesquisa a niños con malnutrición por exceso hacia el odontólogo infantil, con el fin de desarrollar intervenciones preventivas para ambas patologías.

\section{Referencias}

1.- Horowitz HS: Research issues in early childhood caries. Community Dent Oral Epidemiol 1998; 26 (Supl 1): 67-81. 
2.- Milnes AR: Description and epidemiology of nursing caries. J Public Health Dent 1996; 56 (1): 38-50.

3.- Zaror C, Pineda P, Orellana J: Prevalence of early childhood caries and associated factors in 2 an 4 year-old chilean children. Int J Odontostomat 2012; 5: 171-7.

4.- Seow WK: Biological mechanisms of early childhood caries. Community Dent Oral Epidemiol 1998; 26 (Suppl 1): 8-27.

5.- Reisine ST, Psoter $W$ : Socioeconomic status and selected behavioral determinants as risk factors for dental caries. J Dental Educ 2001; 65 (10): 1009-16.

6.- Wigen TI, Espelid I, Skaare AB, Wang NJ: Family characteristics and caries experience in preschool children. A longitudinal study from pregnancy to 5 years of age. Community Dent Oral Epidemiol 2011; 39 (4): 311-7.

7.- Hedley AA, Ogden CL, Johnson CL, Carroll MD, Curtin LR, Flegal KM: Prevalence of overweight and obesity among US children, adolescents, and adults, 1999-2002. JAMA 2004; 291 (23): 2847-50.

8.- Kain J, Uauy R, Vio F, Albala C: Trends in overweight and obesity prevalence in Chilean children: comparison of three definitions. Eur J Clin Nutr 2002; 56 (3): 200-4.

9.- Kain J, Lera L, Rojas J, Uauy R: Obesity among preschool children of Santiago, Chile. Rev Med Chile 2007; 135 (1): 63-70.

10.- Bimstein E, Katz J: Obesity in children: a challenge that pediatric dentistry should not ignore-review of the literature. J Clin Pediatr Dent 2009; 34 (2): 103-6.

11.- Gidding SS, Dennison BA, Birch LL, et al: Dietary recommendations for children and adolescents: a guide for practitioners: consensus statement from the American Heart Association. Circulation 2005; 112 (13): 2061-75.

12.- Dye BA, Shenkin JD, Ogden CL, Marshall TA, Levy SM, Kanellis $M J$ : The relationship between healthful eating practices and dental caries in children aged 2-5 years in the United States, 1988-1994. J Am Dent Assoc 2004; 135 (1): 55-66.

13.- Marshall TA, Eichenberger-Gilmore JM, Broffitt BA, Warren JJ, Levy SM: Dental caries and childhood obesity: roles of diet and socioeconomic status. Community Dent Oral Epidemiol 2007; 35 (6): 449-58.

14.- World Health Organization. Oral health surveys: basic methods. 4th ed. Geneve; 1997.

15.- Greene JC, Vermillion JR: The Simplified Oral Hygiene Index. J Am Dent Assoc 1964; 68: 7-13.

16.- World Health Organization. WHO Child Growth Standards: Length/height-for-age, weight-for-age, weight-for-length, weight-for-height and body mass index-for-age: Methods and development. Geneve, 2006. Dsiponible en: http://www.who.int/childgrowth/ standards/en/.

17.- Zou G: A modified poisson regression approach to prospective studies with binary data. Am J Epidemiol 2004; 159 (7): 702-6.

18.- Pinto A, Kim S, Wadenya R, Rosenberg H: Is there an association between weight and dental caries among pediatric patients in an urban dental school? A correlation study. J Dental Educ 2007; 71(11): 1435-40.

19.- Tenenbaum H, Matthews D, Sandor G, McCulloch C: Oral health-systemic health: what is the true connection? J Can Dent Assoc 2007; 73 (3): 211-6.

20.- Tuomi T: Pilot study on obesity in caries prediction. Community Dent Oral Epidemiol 1989; 17 (6): 289-91.

21.- D'Mello G, Chia L, Hamilton SD, Thomson WM, Drummon $B K$ : Childhood obesity and dental caries among paediatric dental clinic attenders. Int J Paediatr Dent 2011; 21 (3): 217-22.

22.- Chen W, Chen P, Chen SC, Shih WT, Hu HC: Lack of association between obesity and dental caries in threeyear-old children. Zhonghua Min Guo Xiao Er Ke Yi Xue Hui Za Zhi 1998; 39 (2): 109-11.

23.- Macek MD, Mitola DJ: Exploring the association between overweight and dental caries among US children. Pediatr Dent 2006; 28 (4): 375-80.

24.- Gerdin EW, Angbratt M, Aronsson K, Eriksson E, Johansson I: Dental caries and body mass index by socioeconomic status in Swedish children. Community Dent Oral Epidemiol 2008; 36 (5): 459-65.

25.- Alm A: On dental caries and caries-related factors in children and teenagers. Swed Dent J 2008; 195: 7-63.

26.- Sharma A, Hegde AM: Relationship between body mass index, caries experience and dietary preferences in children. J Clin Pediatr Dent 2009; 34 (1): 49-52.

27.- Kantovitz KR, Pascon FM, Rontani RM, Gaviao MB: Obesity and dental caries. A systematic review. Oral Health Prev Dent 2006; 4 (2): 137-44.

28.- Oliveira LB, Sheiham A, Bonecker M: Exploring the association of dental caries with social factors and nutritional status in Brazilian preschool children. Eur J Oral Sci 2008; 116 (1): 37-43.

29.- Li Y, Wang W: Predicting caries in permanent teeth from caries in primary teeth: an eight-year cohort study. J Dental Res 2002; 81 (8): 561-6.

30.- Ribeiro NM, Ribeiro MA: Breastfeeding and early childhood caries: a critical review. J Pediatr (Rio J) 2004; 80 (Suppl 5): S199-210. 